\title{
Analysis of Capacitor Voltage Imbalance in Hybrid Converters and Inherently Balanced Operation Using Symmetric Architecture
}

This paper was downloaded from TechRxiv (https://www.techrxiv.org).

\section{LICENSE}

CC BY-NC-SA 4.0

SUBMISSION DATE / POSTED DATE

02-09-2021 / 09-09-2021

\section{CITATION}

Das, Ratul; Le, Hanh-Phuc (2021): Analysis of Capacitor Voltage Imbalance in Hybrid Converters and Inherently Balanced Operation Using Symmetric Architecture. TechRxiv. Preprint. https://doi.org/10.36227/techrxiv.16557735.v1

$\mathrm{DOI}$ 


\title{
Analysis of Capacitor Voltage Imbalance in Hybrid Converters and Inherently Balanced Operation Using Symmetric Architecture
}

\author{
Ratul Das, Student Member, IEEE, and Hanh-Phuc Le, Senior Member, IEEE
}

\begin{abstract}
This paper investigates the origin of the flying capacitor voltage imbalance in hybrid converters. By observation and logical deduction, an intuitive voltage-charge relationship is established which can give a general explanation of the flying capacitor voltage balance in hybrid converters. This relationship can establish a relatively simple and intuitive method to identify the difference of balance performance in hybrid converters for $V_{\text {out }}<\frac{V_{i n}}{N}$ cases. Converter with even number of inductor charging intervals, are shown to be susceptible to flying capacitor voltage imbalance, while flying capacitors in hybrid converters with inductors having odd charging intervals have inherently balanced operations. As a direct result of the analysis, a new symmetric operation of FCML converters is introduced to achieve an inherent balance of flying capacitor voltages. Hardware implementations and experiments have been carried out for verifications of the analytical analysis and the new symmetric operation.
\end{abstract}

\section{INTRODUCTION}

Hybrid converters have drawn a lot of recent interest from industry and academia because of their benefits in optimizing both passive components, inductors, and capacitors for higher performance operations [1], [2]. Voltage balancing of flying capacitors in hybrid converters is a well-known challenge that attracts a lot of effort in the research community. Most prior publications have largely focused on the phenomena and possible solutions. While effective solutions can be relatively diverse [3], [4], it is important to understand the original mechanism that causes this problem. Using two different approaches, Fourier-based Harmonic analysis in [5] and statespace analysis in [6], it has been shown before that capacitor voltages in an $(\mathrm{N}+1)$-level FCML converter, shown in Fig. 1 becomes exponentially unstable at some nominal conversion ratios. Additional operating states were introduced in [7] to overcome the problems in a 5-level FCML converter. Analytical efforts to explain the problem with practical timing mismatches in FCML converters were also carried out in [8], [9]. The work in [8] numerically shows that odd-level ( $\mathrm{N}$ is even) FCML converters are more sensitive compared to evenlevel ( $\mathrm{N}$ is odd) ones. This analysis is also aligned with the one reported in [10] in terms of identifying the difference of odd and even levels. In [9], the sensitivity of the flying capacitor voltage was calculated for a 3-level buck converter and a 4level Series Capacitor Buck (SCB) Converter.

This paper develops significantly beyond the previous efforts in [8], [9] to investigate a relatively simple procedure to identify the origin of balancing issues and find a solution for inherently balanced operation in FCML converters. The analysis in this paper focuses on the cases with output voltages $V_{\text {out }}$ satisfying $V_{\text {out }}<\frac{V_{\text {in }}}{N}$ compared with the input voltage
$V_{i n}$. The paper starts with the voltage-charge relationship in Section II, which is applied to explain the differences between odd- and even-level FCML converters in Section III. A modified symmetric architecture is proposed in Section IV to achieve inherent balance. Experimental results and verification are included in Section V. The paper is summarized and concluded in Section VI.

\section{Voltage-Charge Relationship}

In simpler power converters, such as Buck, Boost, Cuk, SEPIC, etc., it is possible to determine, with insignificant errors, the steady-state voltages of capacitors in the converter using the Volt-Second balance around the inductor(s). However, for FCML converters, this method does not provide a crucial insight into the flying capacitor voltages. It is required to incorporate the charge balance of the flying capacitor into volt-second balance of the inductors to accurately determine their flying capacitor voltage(s) and draw intuition of the problems for better solutions [9].

In order to accurately determine the capacitor voltages to analyze a possible voltage imbalance scenario, it is critical to monitor the switching node voltages that directly reflects flying capacitor voltages as well as inductor currents. For this goal, we first develop a relationship between the charge flow and switching node voltages in a general FCML converter shown in Fig. 1. This relationship can be intuitively constructed by inductor current because the ripples of inductor current carry the information of the switching node voltages and the output voltage as well as the information of the charges passing through each capacitor.

Figure 2 depicts a general presentation of an inductor current of hybrid converters operating in the inductive region, when $V_{\text {out }}<\frac{V_{i n}}{N} . D_{k-2} T_{S}, D_{k} T_{S}$, and $D_{k+2} T_{S}$ represents three consecutive charging intervals, while $D_{k-1} T_{S}$ and $D_{k+1} T_{S}$ are the intermediate freewheeling intervals when the inductor is connected to ground. Note that, $D_{k-2} T_{S}$ and $D_{k+2} T_{S}$ can also denote the same interval of two consecutive switching periods in a 3-level Buck converter, but they are named separately because of their significance in understanding the general hybrid converters in this operation range.

Assume $Q_{k-2}, Q_{k}$, and $Q_{k+2}$ are the charges through the corresponding voltages at the switching node $V_{x}$ (Fig. 1), $V_{k-2}, V_{k}$, and $V_{k+2}$ during the timing intervals $D_{k-2} T_{S}$, $D_{k} T_{S}$, and $D_{k+2} T_{S}$, respectively. From the median currents and discharging coefficients, $b_{k-2}, b_{k}$ and $b_{k+2}$, previously discussed in [9], a relation among these charges and the 


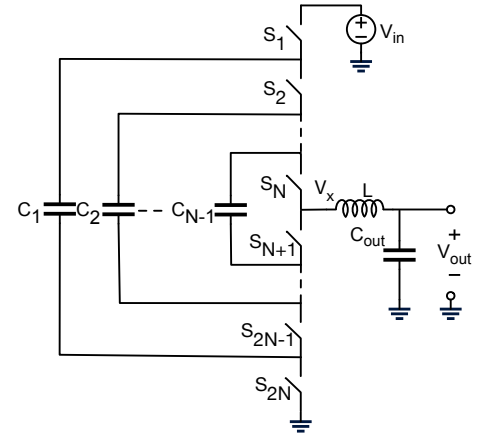

Fig. 1: General schematic of a $(\mathrm{N}+1)$-level FCML converter (A general operation of the FCML converters can be found in [4])

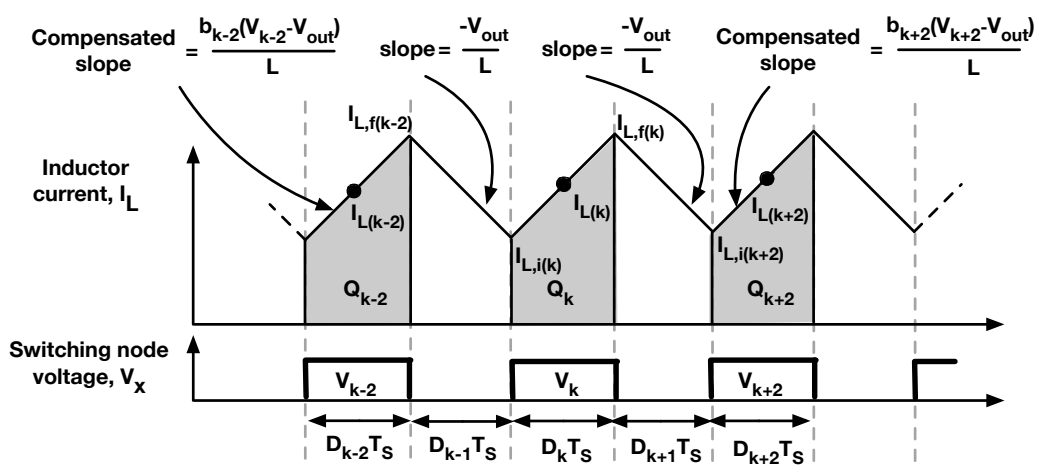

Fig. 2: Inductor current in a FCML converter operating in inductive mode corresponding switch node voltages, $V_{k-2}$ and $V_{k+2}$, can be found as:

$$
\begin{aligned}
& \left(V_{k-2}-V_{\text {out }}\right) \frac{b_{k-2} D_{k-2} T_{S}}{L}-\left(V_{k+2}-V_{\text {out }}\right) \frac{b_{k+2} D_{k+2} T_{S}}{L} \\
& =\frac{2}{T_{S}}\left\{\frac{2 Q_{k}}{b_{k} D_{k}}-\left(\frac{Q_{k-2}}{b_{k-2} D_{k-2}}+\frac{Q_{k+2}}{b_{k+2} D_{k+2}}\right)\right\} \\
& \quad+2 V_{\text {out }}\left(D_{k-1}-D_{k+1}\right) \frac{T_{S}}{L}
\end{aligned}
$$

In Eqn. 1, $V_{\text {out }}$ and $L$ represents the output voltage and inductance. While $b_{k-2}, b_{k}$ and $b_{k+2}$ are very close to 1 in most practical cases, their actual values are important for modeling accuracy. More detailed steps of the derivation of the volt-charge relationship are shown in Appendix A.

The relationship described by Eqn. 1 is general for all stepdown hybrid converters with inductor(s) at the output and freewheeling to the ground, regardless of timing mismatch, voltage imbalances, or other non-idealities in converter operations. A detailed analysis of this relationship can directly explain voltage imbalance behaviors in hybrid converters, as will be shown in the next sections.

Analyzing this relationship inthe ideal match case or in case of small mismatches among parameters of the same type that satisfy the following four conditions: (1) $b_{k-2} \rightarrow b_{k} \rightarrow b_{k+2}$, (2) $D_{k-2} \rightarrow D_{k} \rightarrow D_{k+2}$, (3) $D_{k-1} \rightarrow D_{k+1}$, and (4) $Q_{k-2} \rightarrow Q_{k} \rightarrow Q_{k+2}$, one can find that the two alternate charging voltages converge:

$$
V_{k-2} \rightarrow V_{k+2}
$$

This corollary is true for any alternate charging voltages at $V_{x}$ regardless of their positions in the cycle or across two switching cycles of the converter. While the alternate charging voltages converge, the two consecutive charging voltages at $V_{x}, V_{k-2}$ and $V_{k}$, or $V_{k}$ and $V_{k+2}$, can diverge and become significantly different with capacitor voltage imbalance because of load conditions and various nonidealities, such as input impedance, ESR, parasitic resistance, and timing mismatches, in the circuits. The exact amount of divergence can only be calculated if all these parameters are known. The two consecutive voltages only converge in the

TABLE I: $\mathrm{V}_{\mathrm{x}}$ of 6-level FCML converter

\begin{tabular}{|c|c|c|c|c|c|c|}
\hline States & 1 & 3 & 5 & 7 & 9 & $2,4,6,8,10$ \\
\hline$V_{x}$ & $\mathrm{~V}_{\mathrm{in}}-\mathrm{V}_{\mathrm{C}_{1}}$ & $\mathrm{~V}_{\mathrm{C}_{1}}-\mathrm{V}_{\mathrm{C}_{2}}$ & $\mathrm{~V}_{\mathrm{C}_{2}}-\mathrm{V}_{\mathrm{C}_{3}}$ & $\mathrm{~V}_{\mathrm{C}_{3}}-\mathrm{V}_{\mathrm{C}_{4}}$ & $\mathrm{~V}_{\mathrm{C}_{4}}$ & 0 \\
\hline
\end{tabular}

case of balanced operations. In other words, the $V_{x}$ swing in FCML converter essentially has one of the two voltage levels in the current charging phases of the associate inductor.These two voltage levels are at two consecutive charging phases. They diverge in imbalanced operations but converge in balanced ones. The corollary can be used to understand the voltage imbalance and inductor current fluctuations in FCML converters in the next section and to find a solution to ensure balanced operation.

Equations 1 and 2 are valid for multilevel converters with output voltage, $V_{\text {out }}<\frac{V_{i} n}{N}$, where $\mathrm{N}$ is is number of divisions from the switched capacitor portions.

\section{Application to Multi-Level Converters}

Odd and even-level FCML converters show significantly different balancing performances. In our analysis, we have found out that the reason for this difference is the number of charging phases of the inductor rather than the number of divisions from the switched capacitor levels. Incidentally, even-level $(\mathrm{N}+1)$ FCML converter provides odd number $(\mathrm{N})$ of divisions from the SC structure and odd number of inductor charging intervals. While, odd-level $(\mathrm{N}+1)$ FCML converter has even number $(\mathrm{N})$ of divisions from the SC structure and even number of inductor charging intervals. The balancing performance difference occurs from the number of inductor charging intervals rather than the SC division. This section explains this difference using the voltage-charge relationship and its corollary above for $V_{\text {out }}<\frac{V_{i n}}{N}$.

\section{A. Inductor Current with Odd Number of Charging Intervals}

As an example, Table I lists the switching node voltages at $\mathrm{V}_{\mathrm{x}}$ of a 6-level $(\mathrm{N}=5)$ FCML converter. There are 10 intervals, 5 of which $(1,3,5,7$, and 9) create the rising slopes in the inductor current, while it freewheels from the ground to the output in the remaining states. As analytically proved in the corollary above, there are two sets of voltages at $V_{x}$. Switching node voltages at $\mathrm{V}_{\mathrm{x}}$ during alternate charging intervals 1,5 , and 9 converge to the same value, $V_{i n}-V_{C_{1}}=$

TABLE II: $\mathrm{V}_{\mathrm{x}}$ of 5-level FCML converter

\begin{tabular}{|c|c|c|c|c|c|}
\hline States & 1 & 3 & 5 & 7 & $2,4,6,8$ \\
\hline$V_{x}$ & $\mathrm{~V}_{\text {in }}-\mathrm{V}_{\mathrm{C}_{1}}$ & $\mathrm{~V}_{\mathrm{C}_{1}}-\mathrm{V}_{\mathrm{C}_{2}}$ & $\mathrm{~V}_{\mathrm{C}_{2}}-\mathrm{V}_{\mathrm{C}_{3}}$ & $\mathrm{~V}_{\mathrm{C}_{3}}$ & 0 \\
\hline
\end{tabular}




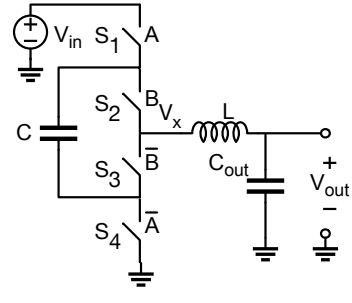

(a) 3-level Buck converter

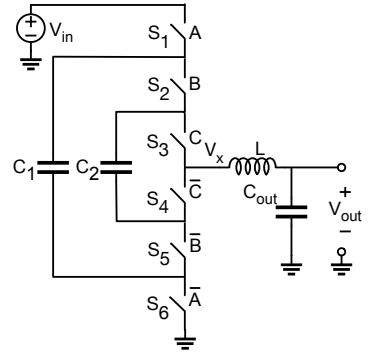

(b) 4-level Buck converter

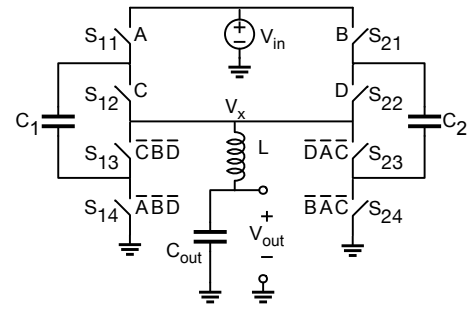

(c) Symmetric 3-level Buck converter for self-balanced operation

Fig. 3: Schematics of odd- and even-level FCML converter examples with annotated switching phases.

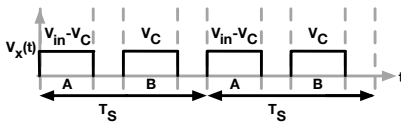

(a) 3-level Buck Converter

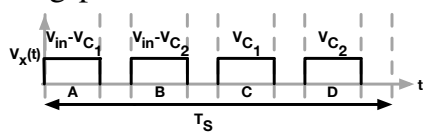

(b) Symmetric 3-level Buck Converter
Fig. 4: Simplified switching node voltages of conventional 3level Buck and a symmetric 3-level Buck converter

$V_{C_{2}}-V_{C_{3}}=V_{C_{4}}$. Similarly, the switching node voltages during interval 3 and 7 also converge, $V_{C_{1}}-V_{C_{2}}=V_{C_{3}}-V_{C_{4}}$. More interestingly, interval 7 of the current switching cycle and interval 1 of the next cycle are also one charging interval (interval 9) apart from each other. From the voltcharge relationship and its corollary above, these two charging voltages also converge, $V_{C_{3}}-V_{C_{4}}=V_{i n}-V_{C_{1}}$. As the result, all the voltages of $\mathrm{V}_{\mathrm{x}}$ during the inductor charging intervals converge to the same value, making a balanced operation. This characteristic is true for all even-level FCML converter ( $\mathrm{N}$ odd) to ensure inherently balanced operations, because they have odd number of inductor charging intervals. However, the same analysis gives a different result for odd-level FCML converters ( $\mathrm{N}$ even).

\section{B. Inductor Current with Even Number of Charging Intervals}

As an example, Table II shows the switching node voltages at $\mathrm{V}_{\mathrm{x}}$ of a 5-level $(\mathrm{N}=4) \mathrm{FCML}$ converter. There are 4 charging intervals $(1,3,5,7)$ and 4 discharging intervals $(2,4,6,8)$ for the inductor current. Switching node voltages at $\mathrm{V}_{\mathrm{x}}$ during intervals 1 and 5 make one converging group, $V_{\text {in }}-V_{C_{1}}=$ $V_{C_{2}}-V_{C_{3}}$, where those of intervals 3 and 7 make another, $V_{C_{1}}-V_{C_{2}}=V_{C_{3}}$. Note that, unlike even-level converter, these two sets of charging voltages do not exchange in the next cycle and nor converge. Therefore, as timing mismatches occur, these two sets of voltages can diverge and cause voltage imbalance in flying capacitors as well as fluctuations in inductor currents. The same characteristic is present in all other odd-level members ( $\mathrm{N}$ even) of the FCML converters that becomes a challenge in fully utilizing the benefits of the FCML architecture.

\section{Balancing Performance Of Other Conventional Hybrid Converters with $V_{\text {out }}<\frac{V_{i} n}{N}$}

Results in subsection III-A and III-B aligns with the results found in [8], [10] for imbalance performance of odd and even level FCML converters, but provides more intuitive approach to understand the imbalance mechanism. The key is in the number of inductor current charging phases and their related switching voltages.

Converters having inductors with an odd number of charging intervals in a switching period, such as even-level FCML converter [1], 6-phase 6-level Dual Inductor Hybrid converter [11], or series capacitor Buck converter [12], [13], have natural balanced operations. The work in [14] demonstrated a hybrid converter with 7 (odd) SC levels and 1 charging interval for each inductor, exhibiting no capacitor voltage imbalance. As another example, the hybrid converter in [11] provides $\frac{1}{6}$ (even-level) to the switching node voltages with each inductor having 3 charging intervals each fundamental switching period, also exhibiting naturally balanced operations.

On the contrary, even number of inductor charging phases causes odd-level FCML converter operation highly susceptible to small timing mismatches and imbalanced capacitor voltages. All traditional odd level FCML converters including 3-level Buck converter fall into this category. 2-level series-capacitor Buck converter [13] with partially coupled inductor also has an even number of inductor charging phases if modeled with a transformer and inductor [15] and hence, it is also susceptible to voltage imbalance. Note that, some other factors, for instance, parasitic resistance and/or hard-charging operation between flying capacitors in higher-level Hybrid Dickson converters [16] or 4-phase 4-level dual inductor hybrid converter [17], can inadvertently minimize imbalance issues at the cost of degraded efficiency.

Note that the analysis and approach in this paper can be extended to other traditional multilevel hybrid converters, for example, the Multi Inductor Hybrid (MIH) converter family [12].

\section{Multi-Phase Symmetric Converter ARCHITECTURE FOR VOLTAGE BALANCING}

Inspired from the analysis above, in this section we propose a new symmetric architecture which can provide natural balanced operation even with an even number of inductor charging intervals, particularly applicable to balance odd-level FCML converter operations. Figure $3 c$ shows a symmetric 3level converter that has two symmetrical SC halves tied to a common switching node with one inductor for soft-charging and output regulation. This converter is operated with four $90^{\circ}$-phased-shifted PWM signals, A,B,C and D, and derived signals $\overline{\mathrm{C}} \overline{\mathrm{B}} \overline{\mathrm{D}}, \overline{\mathrm{A}} \overline{\mathrm{B}} \overline{\mathrm{D}}, \overline{\mathrm{D}} \overline{\mathrm{A}} \overline{\mathrm{C}}$ and $\overline{\mathrm{B}} \overline{\mathrm{A}} \overline{\mathrm{C}}$. This operation also ensures that there is no charge sharing between the two $\mathrm{SC}$ architectures.

Figures $4 \mathrm{a}$ and $4 \mathrm{~b}$ show the switching node voltages of a conventional 3-level Buck and a symmetric 3-level Buck converter. In the conventional 3-level Buck converter, alternate switching node voltages are always generated in the same way, 


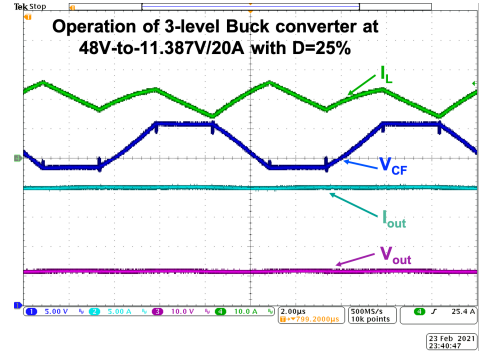

(a) 3-level Buck Converter

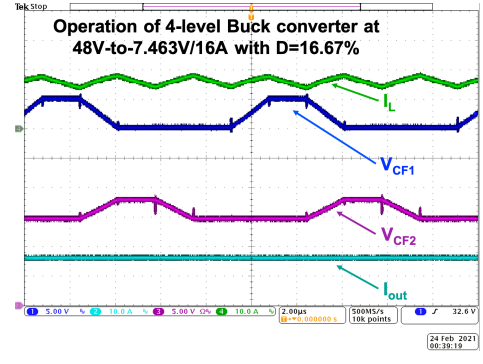

(b) 4-level Buck Converter

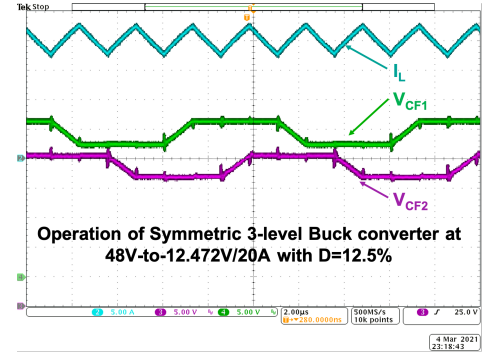

(c) Symmetric 3-level Buck Converter

Fig. 5: Measured waveforms of three hybrid converter prototypes

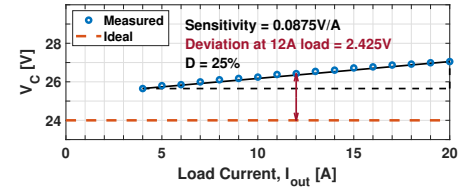

(a) 3-level Buck Converter

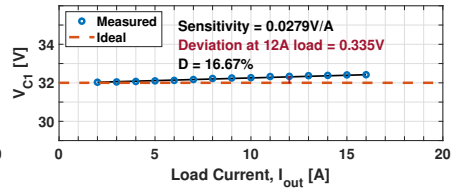

(b) 4-level Buck Converter

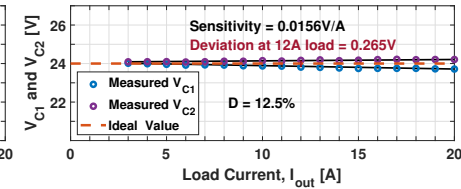

(c) Symmetric 3-level Buck Converter

Fig. 6: Measured median voltages of flying capacitors of hybrid converter prototypes with the same input voltages in Fig. 5.

forming two levels, $V_{i n}-V_{C}$ and $V_{C}$, which are not naturally forced to converge as proved by Eqn. 1 and 2. On the contrary, for the symmetric 3-level Buck converter, the interleaved operation forces $V_{i n}-V_{C 1}$ and $V_{C 1}\left(V_{i n}-V_{C 2}\right.$ and $\left.V_{C 2}\right)$ to converge as they are the alternate switching node voltages. As the result, the flying capacitors have half of the input voltage, $V_{C 1}=V_{C 2}=V_{i n} / 2$, ensuring an inherently balanced operation. Other than generating the right PWM signals, there is no separate control required to tackle timing mismatches or similar non-idealities for balanced operation in this converter . Moreover, the inductor in this converter operates with $4 \times F_{S}$, where $F_{S}$ is the fundamental switching frequency of the converter, where traditional 3-level Buck converter's inductor have twice of the fundamental frequency. This allows the choice of inductance much smaller and thus having benefits from low ESR. Similar symmetric SC structures can be applied to higher odd-level FCML converters to solve a similar voltage imbalance problem.

\section{EXPERIMENTAL VERIFICATION}

To verify the analysis in this paper, a 3-level Buck and a 4-level Buck converter have been implemented to represent odd-level and even-level members of FCML converters with even and odd number of inductor charging shown in Fig. 3a and $3 \mathrm{~b}$ respectively. A symmetric 3-level Buck converter has also been built to verify the proposed inherent balancing. For a fair comparison, all these three converter prototypes use the same type and values of active and passive components and have the same input voltage of $48 \mathrm{~V}$.

The inductor current and flying capacitor voltage waveforms of these three converters are shown in Fig. 5. In Fig. 5a, the 3-level Buck converter exhibits a poor balancing performance, particularly visible in its inductor current. In fact, its balancing problem is also corroborated by its median capacitor voltage shown in Fig. 6a, which deviates from the ideal value of $24 \mathrm{~V}$ and quickly deteriorates with higher currents. Figures $5 \mathrm{~b}$ and $5 \mathrm{c}$ show the waveforms of the 4-level Buck and symmetric 3-level Buck converters, respectively, proving their balanced operation with no apparent visual fluctuation in the inductor currents. Their median capacitor voltages in Figs. 6b and $6 \mathrm{c}$ also reveal much better-balanced operations with $\sim 10 \mathrm{X}$ smaller deviation from the ideal values and up to $\sim 10 \mathrm{X}$ less variation across the load range.

\section{CONCLUSION}

In summary, a new fundamental volt-charge relationship has been devised in this paper which sheds a light on an intuitive analysis of flying capacitor imbalance in hybrid converters for $V_{\text {out }}<\frac{V_{\text {in }}}{N}$ cases. While previous analysis found the difference between odd and even-level flying capacitor multilevel (FCML) converters, the analysis in the paper shows that the reason for their difference is the even and odd number of inductor charging intervals. This simplifies the method to identify balanced or imbalanced characteristics in traditional hybrid converters by only counting the number of inductor charging intervals within a fundamental switching period. The experimental results for verification of the analysis is conveyed with 3 and 4-level FCML converters. A new symmetric architecture has also been proposed, implemented, and verified for a 3-level Buck converter to show that hybrid converters with even number of inductor charging intervals can be made naturally balanced with an appropriate architecture and operation. The analysis, method for identifying imbalanced converters, and the proposed symmetric architecture as a solution can be extended to other hybrid converters.

\section{REFERENCES}

[1] C. Schaef et al., "A 93.8\% Peak Efficiency, 5V-Input, 10A Max ILOAD Flying Capacitor Multilevel Converter in 22nm CMOS Featuring Wide Output Voltage Range and Flying Capacitor Precharging," in 2019 IEEE Int. Solid- State Circuits Conf., Feb. 2019, pp. 146-148.

[2] C. Hardy et al., "A Flying-Inductor Hybrid DC-DC Converter for 1-Cell and 2-Cell Smart-Cable Battery Chargers," IEEE J. Solid-State Circuits, vol. 54, no. 12, pp. 3292-3305, Dec. 2019.

[3] A. Stillwell et al., "Active Voltage Balancing in Flying Capacitor MultiLevel Converters With Valley Current Detection and Constant Effective Duty Cycle Control," IEEE Trans. Power Electron., vol. 34, no. 11, pp. $11429-11441$, Nov. 2019. 
[4] J. S. Rentmeister and J. T. Stauth, "A 48V:2V flying capacitor multilevel converter using current-limit control for flying capacitor balance," in 2017 IEEE Appl. Power Electron. Conf. and Expo., Mar. 2017, pp. 367372.

[5] R. H. Wilkinson et al., "Natural Balance of Multicell Converters: The General Case," IEEE Trans. Power Electron., vol. 21, no. 6, pp. 1658 1666, Nov. 2006.

[6] Z. Xia et al., "State Space Analysis of Flying Capacitor Multilevel DCDC Converters for Capacitor Voltage Estimation," in 2019 IEEE Appl. Power Electron. Conf.(APEC)), Annaheim, Los Angeles, Mar. 2019.

[7] — "Natural Balancing of Flying Capacitor Multilevel Converters at Nominal Convers. Ratios," in 2019 20th Workshop Control Model. Power Electron., Jun. 2019, pp. 1-8.

[8] J. Celikovic et al., "Modeling of Capacitor Voltage Imbalance in Flying Capacitor Multilevel DC-DC Converters," in 2019 20th Workshop Control Model. Power Electron., Jun. 2019, pp. 1-8.

[9] R. Das et al., "Demystifying Capacitor Voltages and Inductor Currents in Hybrid Converters," in 2019 20th Workshop Control Model. Power Electron., Jun. 2019, pp. 1-8.

[10] Z. Ye et al., "Investigation of capacitor voltage balancing in practical implementations of flying capacitor multilevel converters," in 2017 IEEE 18th Workshop Control and Model. Power Electron. (COMPEL), Jul. 2017, pp. 1-7.

[11] T. Xie et al., "Multiphase Control for Robust and Complete Softcharging Operation of Dual Inductor Hybrid Converter," in 2019 IEEE Appl. Power Electron. Conf. and Expo., Mar. 2019, pp. 1-5.

[12] R. Das and H.-P. Le, "A Regulated 48V-to-1V/100A 90.9\%Efficient Hybrid Converter for POL Applications in Data Centers and Telecommunication Systems," in 2019 IEEE Appl. Power Electron. Conf. and Expo., Mar. 2019, pp. 1997-2001.

[13] P. S. Shenoy et al., "Comparison of a Buck Converter and a Series Capacitor Buck Converter for High-Frequency, High-Convers.-Ratio Voltage Regulators,' IEEE Trans. Power Electron., vol. 31, no. 10, pp. 7006-7015, Oct. 2016.

[14] R. Das et al., "Analysis of Dual-Inductor Hybrid Converters for Extreme Convers. Ratios," IEEE J. Emerg. Sel. Top. Power Electron., pp. 1-1, 2020.

[15] P. Yang et al., "Performance improvements of interleaving VRMs with coupling inductors," IEEE Trans. Power Electron., vol. 16, no. 4, pp. 499-507, Jul. 2001.

[16] Y. Lei et al., "A GaN-based 97\% efficient hybrid switched-capacitor converter with lossless regulation capability," in 2015 IEEE Energy Convers. Congr. and Expo., Montreal, QC, Sep. 2015, pp. 4264-4270.

[17] S. Khatua et al., "A Non-Isolated Single-Stage 48V-to-1V VRM with a Light Load Efficiency Improvement Technique," in 2018 IEEE Energy Convers. Congr. and Expo., Sep. 2018, pp. 143-148.

\section{APPENDIX A}

\section{DERIVATION OF VOLTAGE-CHARGE RELATIONSHIP}

Voltage-charge relationship can be derived from the relationships of median currents $I_{L(k-2)}, I_{L(k)}$ and $I_{L(k+2)}$ of the inductor in charging intervals $D_{k-2} T_{S}, D_{k} T_{S}$ and $D_{k+2} T_{S}$ in Fig. 2. If the peak currents of the intervals $D_{k-2} T_{S}$ and $D_{k} T_{S}$ are $I_{L, f(k-2)}$ and $I_{L, f(k)}$, and the valley currents of the intervals $D_{k} T_{S}$ and $D_{k+2} T_{S}$ are $I_{L, i(k)}$ and $I_{L, i(k+2)}$, it can be written that,

$$
\begin{aligned}
& I_{L(k)}=\frac{I_{L, i(k)}+I_{L, f(k)}}{2} \\
& =\frac{\left\{I_{L, f(k-2)}-\frac{V_{\text {out }}}{L} D_{k-1} T_{S}\right\}+\left\{I_{L, i(k+2)}+\frac{V_{\text {out }}}{L} D_{k+1} T_{S}\right\}}{2} \\
& =\frac{\left(I_{L(k-2)}+\frac{b_{k-2}\left(V_{k-2}-V_{\text {out }}\right)}{2 L} D_{k-2} T_{S}\right)-\frac{V_{\text {out }}}{L} D_{k-1} T_{S}}{2} \\
& \quad+\frac{\left(I_{L(k+2)}-\frac{b_{k+2}\left(V_{k+2}-V_{\text {out }}\right)}{2 L} D_{k+2} T_{S}\right)+\frac{V_{\text {out }}}{L} D_{k+1} T_{S}}{2} \\
& =\frac{I_{L(k-2)}+I_{L(k+2)}+\frac{T_{S}}{4 L}\left\{b_{k-2} D_{k-2}\left(V_{k-2}-V_{\text {out }}\right)\right.}{2} \\
& \left.\quad-b_{k+2} D_{k+2}\left(V_{k+2}-V_{\text {out }}\right)\right\}-V_{\text {out }} \frac{T_{S}}{2 L}\left(D_{k-1}-D_{k+1}\right)
\end{aligned}
$$

If we replace $I_{L(k)}=\frac{Q_{k}}{b_{k} D_{k} T_{S}}, I_{L(k-2)}=\frac{Q_{k-2}}{b_{k-2} D_{k-2} T_{S}}$ and $I_{L(k+2)}=\frac{Q_{k+2}}{b_{k+2} D_{k+2} T_{S}}$ in Eqn. 3, it can be rewritten as,

$$
\begin{aligned}
\frac{Q_{k}}{b_{k} D_{k} T_{S}}= & \frac{1}{2}\left(\frac{Q_{k-2}}{b_{k-2} D_{k-2} T_{S}}+\frac{Q_{k+2}}{b_{k+2} D_{k+2} T_{S}}\right) \\
& +\frac{T_{S}}{4 L}\left\{b_{k-2} D_{k-2}\left(V_{k-2}-V_{\text {out }}\right)\right. \\
& \left.-b_{k+2} D_{k+2}\left(V_{k+2}-V_{\text {out }}\right)\right\} \\
& -V_{\text {out }} \frac{T_{S}}{2 L}\left(D_{k-1}-D_{k+1}\right)
\end{aligned}
$$

Eqn. 4 can be re-arranged as volt-charge relationship in Eqn. 